\title{
CORRECTING GARMENT SET DEFORMALITIES ON VIRTUAL HUMAN MODEL USING TRANSPARANCY MEASURES
}

\author{
R. Anil Kumar ${ }^{1}$, R.I. Sathya ${ }^{2}$ \\ ${ }^{I}$ Full Time Ph.D Research Scholar, Department of Home Science, Gandhigram Rural Institute-Deemed University, Tamil \\ Nadu, India \\ ${ }^{2}$ Associate Professor, Department of Home Science, Gandhigram Rural Institute-Deemed University, Tamil Nadu, India
}

\begin{abstract}
Designing has changed it dimensions from 2D, 3D to real time simulation and virtual designing environment. This study is about correcting the garment set deformalities in designing that occurs mainly due to the solid object overlapping method which causes the base model to be hidden. This is applicable to the edges, seam positions, inseam positions, hem line placement and many other areas. To overcome the offsets in these areas transparency based measures has to be adopted from the designing phase itself. This method ensures a well set garment to the exact seam position and parts alignment over the model. This method is applicable to both $2 D$ and $3 D$ designing environment. The level of transparency depends on the visibility of the background model. This method overcomes many difficulties that occur while designing based on a solid object method.
\end{abstract}

Keywords: Transparency, Opacity, Set, Model, Simulation, Design

\section{INTRODUCTION}

Apparel designing has undergone tremendous technological and scientific up gradations from the past decades. Computers have been an unavoidable device for pattern making, grading, embroidery designing, screen print designing, design creation systems and manipulation system. Developing a virtual model involves intricate job like adding skin tone to the desired race, adding shade to the skin, accurate body part joints, and controlling the vertices on cloth parts as well as body parts, accuracy in structuring and interpretation of the cloth parts to the virtual model, application of texture to the garmented surface and controlling the mesh resolution [1]. The garments are fitted on to a virtual body by means of a physical simulation technique. The virtual environment requires complex understanding and skills in modeling and is more widely suitable for fashion designers, animators, film developers and game developers. Implementing these tasks requires a lot of design time but these creation of these realistic virtual characters in a virtual environment also requires computation skills. Modeling is classified into two groups; structural and mathematical [1] [3].The structural based designing aspect requires anthropometric knowledge which is used to organize and construct virtual models. In mathematical based designing physical or geometrical aspects are used to represent the virtual model [2].

Cloth modeling tool are used in the apparel manufacture to design and sample garments before the bulk production process. These modeling tools fit into the needs of the industry requirement, targeting on fabric simulation and fit analysis. Clothing simulation tools helps the designers to create 3D clothes on a 3D human model. The garment must be physically simulated based on accurately measured physical parameters to allow the evaluation of dynamic garment behavior to fitting. $2 \mathrm{~d}$ and $3 \mathrm{D}$ cloth simulation uses a draping/simulation $3 \mathrm{~d}$ engine that allows the user to see how the final product fits on a body with certain dimensions. The fitting of the garment is affected by the visual appearance and not by the fit to a certain size [4]. All possibilities and parameters depending on the level of abstractness can be imagined which forces the surfaces to approach along the seam lines. The simulation is executed until the fabric is dynamically stabilized in a static position.

A garment is said to be of well set when it doesn't have any undesirable wrinkles and crease marks.

Transparency is a method of revealing image areas underneath the object. Transparency may be uniformly set, linear, radical, conical, square, two colored pattern, full colored pattern, bitmap pattern, texture base transparency. There are several methods of applying transparency to a object like normal, add, subtract, difference, multiply, divide, lighter, darker, texturizing, hue, saturation, lightness, invert based transparency etc. transparency can be set to freeze that is by freezing the current view of the object through the transparency so that the view does not change when the object is moved. The benefits in using a transparency option is that the set transparency value attributes can be copied from the 
selected object to another object in the document to the selected object. One can also remove the transparency from the object [5] [6].

Setting the designing nodes to the exact position requires specific technical skill. A solid object based designing doesn't provide any visible background region on the surface and hence the inset object placement is difficult. Matching of checked patterns, plaids or striped designs are difficult in solid object designing.

The analysis of designs based on transparency object modeling over a virtual model and correcting the set deformalities in positioning of seams and garment parts is discussed in this study.

\section{EXPERIMENTAL}

\subsection{Developing a Child Mesh Model}

The wireframe human model is developed using Make Human 1.0.0 open source software for making 3D human characters which is used for Real Time Simulation. This is a user friendly application which provides easier modeling facility in a mesh surface. The virtual human characters can be viewed from any angle and the characters can be rendered creating using Make Human software can be manipulated and viewed in any angle with the help of an inbuilt camera feature within the application. The built in camera feature allows capturing the screen image and storing the captured image in the desired location.

Table - 1: Measurement chart of Virtual Child Mesh Model

\begin{tabular}{|l|l|l|l|}
\hline $\begin{array}{l}\text { S. } \\
\text { No }\end{array}$ & \multicolumn{2}{|c|}{ Feature } & \multirow{2}{*}{ Virtual Child Model } \\
\hline 1 & Gender & Male \\
\hline 2 & Age & 10 \\
\hline 3 & Muscle & $50 \%$ \\
\hline 4 & Weight & $100 \%$ \\
\hline \multicolumn{3}{|l|}{ Measurements (in cm) } \\
\hline 1 & Height & 138 \\
\hline 2 & Chest & 78 \\
\hline 3 & Waist & 61 \\
\hline 4 & Hip & 73 \\
\hline 5 & Neck Round & 31.5 \\
\hline 6 & Neck height & 9.2 \\
\hline 7 & $\begin{array}{l}\text { Upper arm } \\
\text { circ }\end{array}$ & 20 \\
\hline 8 & $\begin{array}{l}\text { Upper arm } \\
\text { length }\end{array}$ & 22.6 \\
\hline 9 & $\begin{array}{l}\text { Lower arm } \\
\text { length }\end{array}$ & 19 \\
\hline 10 & Wrist circ & 61 \\
\hline 11 & Front chest & 24.6 \\
\hline
\end{tabular}

\begin{tabular}{|l|l|l|}
\hline & dist & \\
\hline 12 & $\begin{array}{l}\text { Nape to } \\
\text { waist }\end{array}$ & 30 \\
\hline 13 & Waist to hip & 15 \\
\hline 14 & $\begin{array}{l}\text { Shoulder } \\
\text { dist }\end{array}$ & 10.3 \\
\hline 15 & $\begin{array}{l}\text { Upper leg } \\
\text { height }\end{array}$ & 27.2 \\
\hline 16 & Thigh circ & 41 \\
\hline 17 & Lower leg ht & 41.2 \\
\hline 18 & Calf circ & 30.2 \\
\hline 19 & Ankle circ & 18.3 \\
\hline
\end{tabular}

The Table 1 shows the Measurement chart for the Virtual Child model. There are 19 important measurements that are required to construct the Virtual model with Make Human 1.0.0. A model for 10 year old boy was developed using the standard measurement chart.

\subsection{Rendering}

The Make Human 1.0.0 provide wide rendering option like Blender exchange (.mhx), Film box (.fbx), Collada (.dae), wave front object, MD5, Ogre 3D, Stereo lithography (.Stl), Biovision Hierarchy BVH, Skeleton (skel), Jpeg, Multipicture (.mpo), Pixus (.pcx), Scitex (.sct). The virtual human mesh model was rendered in jpeg format for 2D designing, simulation and visualization.

\subsection{Solid Object Modeling}

A solid object modeling is a critical modeling system as the solid base object layer hides the background object. It is difficult to set the object to the exact outline of the background object. In designing the task of solid based modeling is setting the layer object on line with the background object. Solid modeling can be done on plain color, fountain filled, texture filled, pattern filled or post script filled objects. 


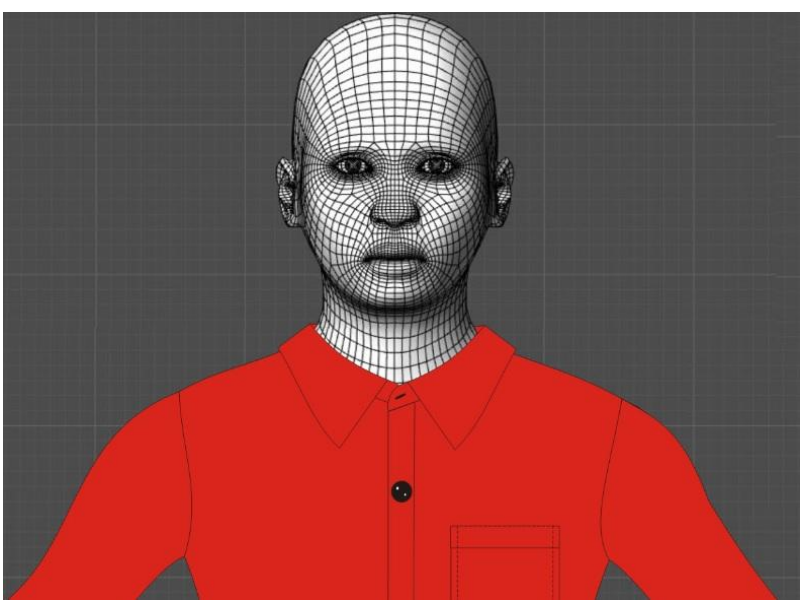

Fig - 1: Virtual child mesh model Solid base Garmenting

The Figure 1 shows the Virtual Child mesh model solid object garmenting over the mesh model using Corel Graphics Suite X5. The solid object over the mesh model hides the background path of the mesh model.

\subsection{Transparency Based Object Modeling}

A transparency based object modeling is an easier modeling system compared to solid based object modeling as the base object doesn't hide the background object. It is easier to set the base object to the exact outline and seam position of the background object. Transparency can be set to any solid form of object as plain color, fountain filled, texture filled, pattern filled or post script filled objects. Apart from setting the points to exact seam matching of checks, stripes or plaids can be easily done by this method.

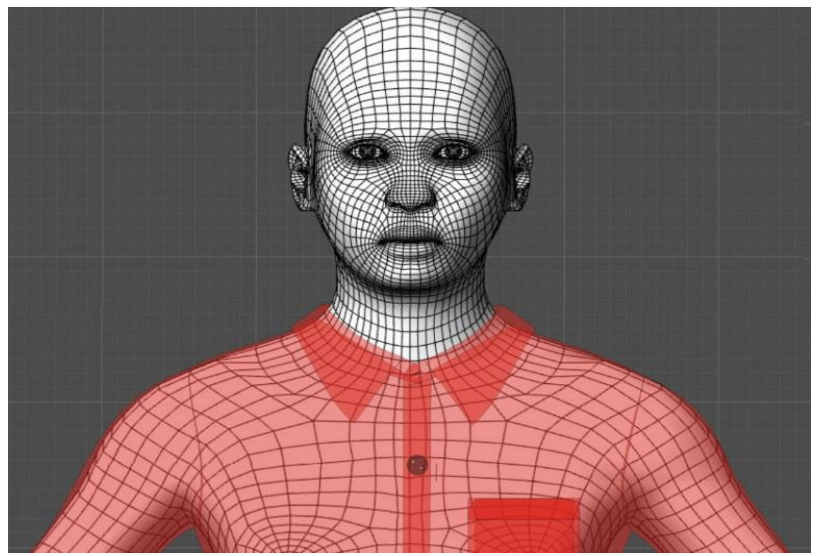

Fig - 2: Virtual child mesh model Transparency base Garmenting

The Figure 2 shows the Virtual Child mesh model transparency based garmenting over the mesh model using Corel Graphics Suite X5. The transparent object over the mesh model shows the background mesh model as well as the grid positions very clearly hence it is easier to plot node marks at the seam positions.

\section{RESULTS AND DISCUSSION}

A complete transparency based modeling over a mesh kid model has been demonstrated below.

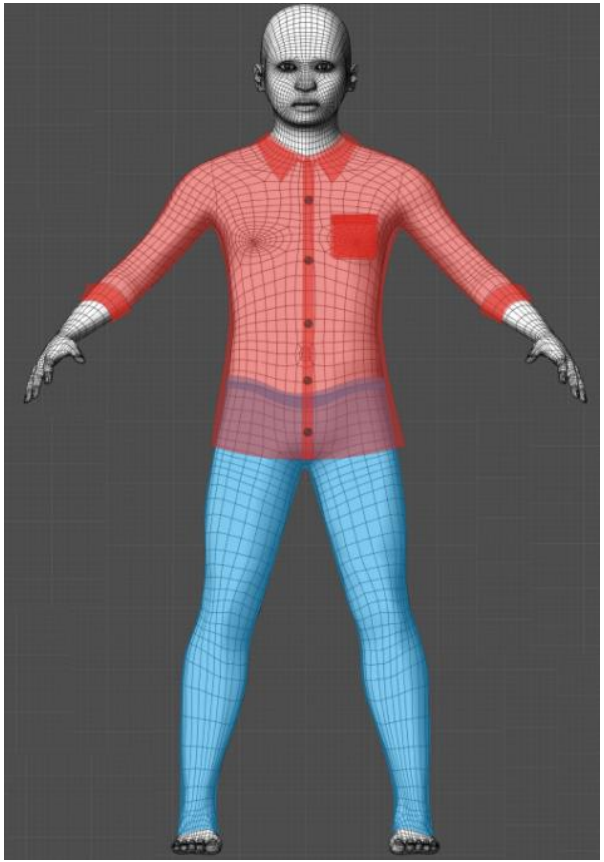

Fig - 3: Virtual child mesh model Transparency base Front image

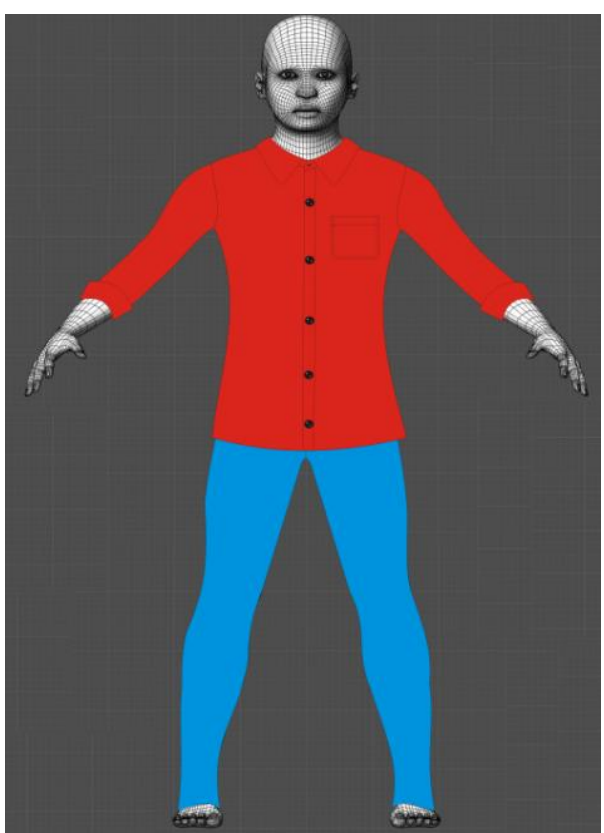

Fig -4: Virtual child mesh model Solid base Front image 


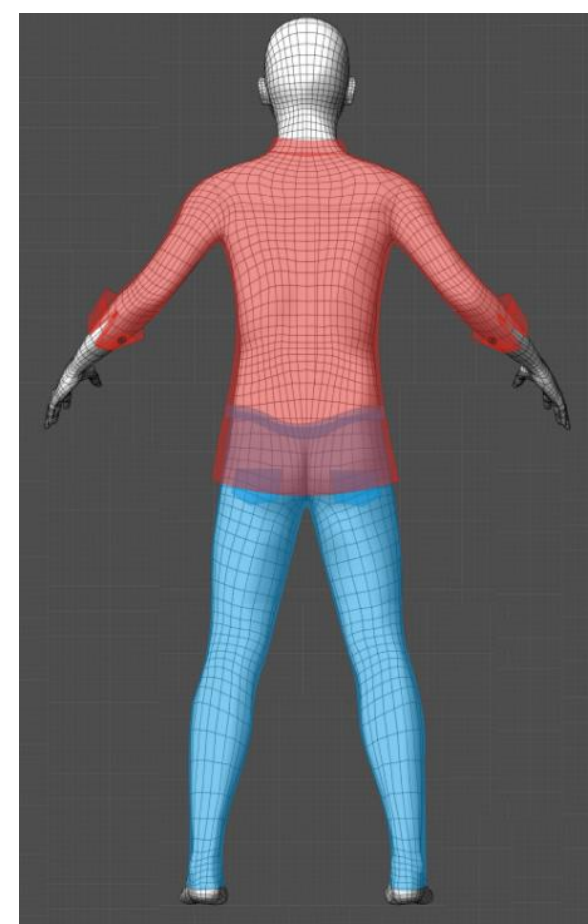

Fig - 5: Virtual child mesh model Transparency base back part

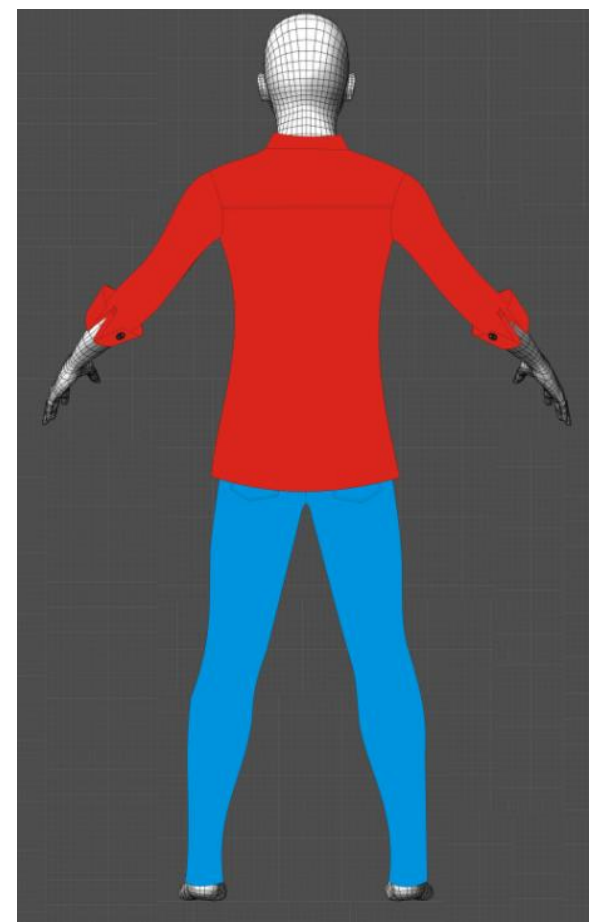

Fig - 6: Virtual child mesh model Solid object back part

The Figure 3 and Figure 4 shows the Virtual Child mesh model transparency based garmenting and virtual mesh solid object modeling. Figure 3 clearly shows the background grid which is used for garmenting the model. Figure 4 is the solid complete image where the transparency has been removed.
Figure 5 shows the virtual child mesh models back view which was drafted on transparency based modeling. Figure 6 is the solid image of Figure 5 where the transparency has been removed. The solid images clearly hide the background mesh and solid modeling is a difficult process.

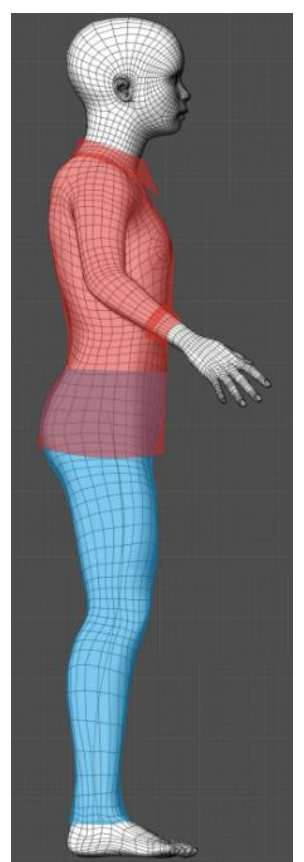

Fig - 7: Virtual child mesh model transparent side view

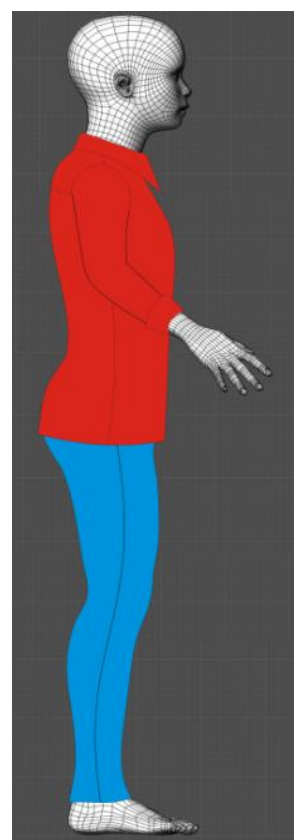

Fig - 8: Virtual child mesh model Solid base side view

The Figure and Figure 8 shows the Virtual child transparent based side view and solid base side view. 


\section{CONCLUSIONS}

A detailed analysis of the designing based on Transparency method the designing based on solid object method it is very clear that transparency method is the most accurate method as the background image is very clearly visible in this method. This is applicable to the edges, seam positions, inseam positions, hem line placement and many other areas. To overcome the offsets in these areas transparency based measures has to be adopted from the designing phase itself. This method ensures a well set garment to the exact seam position and parts alignment over the model. This method is applicable to both $2 \mathrm{D}$ and $3 \mathrm{D}$ designing environment. The level of transparency depends on the visibility of the background model. This method overcomes many difficulties that occur while designing based on a solid object method. A solid object based designing doesn't provide any visible background region on the surface and hence the inset object placement is difficult. Matching of checked patterns, plaids or striped designs are difficult in solid object designing.

\section{REFERENCES}

[1]. Baraff .D, Witkinv.A, (1998) Large steps in cloth simulation. ACM SIGGRAPH, pp. 43 - 54.

[2]. Choi K.J., Ko K.S., (2002) Stable but responsive cloth. ACM SIGGRAPH.

[3]. Barr A. (1984) Global and local deformations of solid primitives. SIGGRAPH '84, Proceedings of the 11th Annual Conference on computer graphic and interactive techniques (pp. 21 - 30). ACM Press.

[4]. Allen .B, Curless .B, Popovic .Z, (2003) The space of human body shapes: Reconstruction and parameterization from range scans. ACM Transactions on Graphics 22, pp. 587 $-594$.

[5]. Thalmann, Nadia Magnenat (2010) Modeling and Simulating Bodies and garments. London: springer Verlag .

[6]. Terzopoulos .D, Platt .J, Barr .A, Fleischer .K, (1987) Elastically Deformable models. SIGGRAPH '84, Proceedings of the 14th Annual Conference on computer graphics and Interactive techniques (pp. 205 - 214). ACM Press. 